\title{
Correspondence
}

Editor: Ian Pullen

Contents: Oedipus at Delphi/Clozapine and negative symptoms/Neuroleptic rechallenge after neuroleptic malignant syndrome/Salivary testosterone levels and major depressive illness in men/Attitudes to mental illness/An Eysenck for the 1990s?/Integration of psychodynamics in Scandinavian psychiatry/Longterm antidepressant treatment in the elderly/Child psychiatric syndromes with a somatic presentation/ Distribution of adipose tissue in patients receiving psychotropic drugs/Parental bonding/The influence of ethnicity and family structure on relapse in firstepisode schizophrenia/Tattooed female psychiatric patients/Postage stamp test for sexual disorders/ Gastric emptying and bloating in anorexia nervosa/ Blood-letting in bulimia nervosa/Abuse of the nasogastric tube in patients with eating disorders.

\section{Oedipus at Delphi}

SIR: Pharmaceutical companies are businesses. The NHS, at present, is not. Therein lies a potential for ethical dilemma. To argue that noting this is tantamount to accusing companies ipso facto of white collar crime (Bosanquet \& Zajdler, Journal, January 1993, 162, 29-32) seems remarkable.

The primary 'ethic' for a pharmaceutical company is survival, in the pursuit of which Sandoz has set the price of clozapine at a different order of magnitude to that of any other psychotropic drug. This price covers development, liability, and marketing costs, but is also set to yield appreciable profit and is set in great part by a company's perception of what the market will bear. The emergence of budget-busting compounds shows that in certain circumstances the market will bear considerably more than was otherwise thought possible.

In the case of clozapine, it could be argued that the price must be set high as its distribution would be limited to a small population of treatment-resistant patients. However, it could also be expected that aggressive marketing on the part of the company, pressure from patients and relatives for access to this 'wonder' drug, featured on the front cover of Time magazine, and the clinical ethic of offering what is perceived to be the best possible care to each individ- ual who seeks our help, would see to it that far more individuals would receive clozapine than might have been initially expected, given the terms of its license.

There are a number of implications of recent developments in neuroleptic psychopharmacology, including the Kane et al (1988) study, that many prescribers may not appreciate when considering a patient for treatment with clozapine. One is that before concluding that a patient is resistant to treatment, a sustained course of low dose chlorpromazine (c. $50 \mathrm{mg} /$ day) should be tried - sustained for up to six or nine months to judge by current (Sandoz) recommendations for clozapine. There are good grounds for suggesting that a stimulant should also be tried (Lieberman et al, 1987). How many clinicians try all these kind of changes before concluding that the subject is treatment resistant?

There are also notable hidden costs to clozapine prescribing that are not addressed by Davies \& Drummond (Journal, January 1993, 162, 38-42). The health authority where I work has put a ceiling of six on the number of patients who may be on clozapine at one time. Currently, all six live in a catchment area (55000 patients) which I cover with a colleague, and five are in my care. One is on clozapine because of a remarkable propensity to extrapyramidal sideeffects from other neuroleptics. Two have chronic schizophrenia and two chronic delusional disorder. Of these five patients, four are calmer than they were before starting clozapine but no less deluded or thought disordered; the fifth has fewer extrapyramidal symptoms but is otherwise no different than if on another neuroleptic. Four of the five patients lived at home in the first instance and hence there are no savings accruing from discharge from institutional care. In this catchment area, there is only one patient in high-cost, institutional care who could be expected to leave care if responsive to clozapine - she was not. Even if more fully responsive to clozapine, none of the six patients could expect to return to gainful employment.

The population in this catchment area is dispersed, so that taking blood from these six patients (to check for agranulocytosis, which the drug may cause), and delivering pills to them, requires $50 \mathrm{~km}$ per patient 
per week. If we add to travelling time the time spent on the telephone liaising between Sandoz and three hospital pharmacies, as is required in this area, the total 'dead' time that would otherwise be given to patient care amounts to half of a full-time equivalent $F$ grade community psychiatric nurse $(\mathrm{CPN})(£ 8400)$

Given the price of the next most expensive psychotropic compounds, the 5-HT reuptake inhibitors, is $£ 400$ per year, we can add $£ 1600$ per patient to this bill to represent the excess cost specifically accruing from clozapine. In this catchment area, this will then amount to a further $£ 9600$.

In the course of keeping these six patients on clozapine, owing to inflexibility on the part of the company as to when the blood samples are taken, CPNs have regularly had to miss ward rounds, team meetings and out-patient sessions. The opportunity costs of not having a patient's keyworker at one of the above settings can be estimated at $30 \%$ of 20 (members of the team) times $£ 10$ (mean hourly wage) times 4 (hours per notional half day per week), which comes to $£ 12400$ per year.

Opportunity costs could be reduced by the entire team restructuring its activities to conform with the needs of Sandoz or the provision of a dedicated clozapine nurse. Economies could also be achieved by more patients being put on clozapine.

In some teams the costs may be less if the taking of blood samples from clozapine-treated patients simply replaces the giving of a depot neuroleptic. In this team at present, a number of CPNs are participating in a research project looking at the efficacy of cognitive therapy for patients with chronic neuroleptic refractory delusional states (Lowe \& Chadwick, 1990), and another project is commencing aimed at establishing groups to manage auditory hallucinations employing the techniques outlined by Romme et al (Journal, July 1992, 161, 99-103). If all the extra costs of clozapine could be translated into CPN time, or social worker time, up to 1500 hours per year of innovative face-to-face mental health work with 'neuroleptic'-resistant patients of these kinds could be realised, in this area alone.

As regards Delphi panels, emergent views will be shaped by the constitution of the panel. There is at present no proof of the objectivity or validity of the judgements reached by a panel, such as that employed by Davies \& Drummond. Ideally, a panel should have a balance of sceptics and enthusiasts, and the methods section of a paper should detail the recruitment process. In the case of an issue such as this there perhaps should also be a balance between those who manage large unreconstructed rehabilitation services and those who do not. There should be some guidelines on the affiliations or links panellists may have with any company involved and the independence of the data to which appeals are made, or else either some method for taking these into account or a statement to alert the reader. Failure to take account of these issues is all too likely to leave any claims open to criticism, for example by Breggin (1991).

In comparing the clozapine phenomenon to that of insulin coma, I was far from wishing to suggest that clozapine does not work (Kerwin, Journal, April $1993,162,566-567)$. The point rather was that insulin and the enthusiasm associated with its use quite clearly did work. Authoritative or oracular pronouncements encourage the rest of us to try things we might not otherwise have done (Oedipus effect). Assessing the effects of psychotropic drugs by a Delphi panel offers a potent method to procure Oedipal effects. At what cost to our eyesight?

I agree with Bosanquet \& Zajdler that new drugs would represent innovation in mental health work, but would suggest that there have been no radically new psychotropic compounds synthesised since the mid-1960s; which may account in part for an increased recourse to health economics (see Luke effect; Healy, 1990). I also agree that close relations between 'professionals' and the pharmaceutical industry would increase the chances of new development, but would argue that currently this is more frustrated by an inherent conservatism on the part of the industry, whose requirements for safe profits are putting increasing constraints on drug development programmes, than it is by any conservatism on the part of clinicians.

In business, the dominant ethic is one of seeking a profit in order to develop. This usually involves risk. In health care, there is a need to include the community, who are the primary risk takers, in any strategy. The strategy pharmaceutical companies adopt to manage this risk has varied historically (Lieberman et al, 1987). The benzodiazepine saga remains as a recent example of how companies can differ in their strategies, in a manner that leads to the prescription of a therapeutically useful group of compounds, to the detriment of other companies and of the mentally ill.

Breggin, P. R. (1991) Toxic Psychiatry. New York: St Martin's Press.

Healy, D. (1990) The psychopharmacological era: notes toward a history. Journal of Psychopharmacology, 4, 152-167.

KANE, J., Honigreld, G., Singer, J., et al (1988) Clozapine for the treatment-resistant schizophrenic. Archives of General Psychiatry, 45, 789-796.

Lieberman, J. A., Kane, J. M. \& Alvir, J. (1987) Provocative tests with psychostimulant drugs. Psychopharmacology, 91, 415-433. 
LOWE, C. F. \& CHADWICK, P. J. (1990) Verbal control of delusions. Behaviour Therapy, 21, 461-480.

David Healy Academic Subdepartment of Psychological Medicine Denbigh, Clwyd LL16 5SS

\section{Clozapine and negative symptoms}

SIR: I read with interest about the remarks made by Healy (Journal, January 1993, 162, 23-29) on the superiority of clozapine over traditional neuroleptics and would like to express some reservations about this drug's ability to improve the negative symptoms of schizophrenia. Negative symptoms are ill-defined, and lack firmly established construct validity; they may be mimicked by drug-induced akinesia and depression (de Leon, 1989). The apparent improvement of negative symptoms in Kane et als (1988) study may be for a number of reasons. These include the reduction of positive symptoms, the use of a nonspecific measurement scale (Brief Psychiatric Rating Scale), the relief of depression in chronic psychotic patients (clozapine is suspected of having some antidepressant property), and diminution of extrapyramidal side-effects. The latter possibility was supported by the significantly lower scores for extrapyramidal side-effect of the clozapine group than the chlorpromazine group from week four until the end of the trial. Perhaps a better way to prove clozapine's superiority in improving negative symptoms would be a controlled trial of treatment for 'pure negative syndrome', simple schizophrenia, or the residual negative syndrome. Until then we should be sceptical of its specificity in improving negative symptoms.

DE LeON, J., Wilson, W. H. \& Simpson, G. M. (1989) Measurement of negative symptoms in schizophrenia. Psychiatric Development. 3, 211-234.

KANe, J., Honigfeld, G., Singer, J., et al (1988) Clozapine for the treatment-resistant schizophrenic. Archives of General Psychiatry, 45, 789-796.

Prince of Wales Hospital

YUN-KwoK WING

Chinese University of Hong Kong

Shatin, NT

Hong Kong

\section{Neuroleptic rechallenge after neuroleptic malignant syndrome}

SIR: We read with interest the report by Weller \& Kornhuber (Journal, December 1992, 161, 855-856) on the use of the atypical antipsychotic clozapine after an episode of neuroleptic malignant syndrome (NMS). Being also concerned with the clinical man- agement and follow-up of this syndrome, we feel it necessary to raise some points regarding the question of whether and which neuroleptic drug could be readministered as a further treatment of psychotic symptoms.

We would like to emphasise that the expression 'clozapine rechallenge' which is notified in the title of the article could lead to a potentially harmful misinterpretation. Indeed, the term rechallenge is defined as the giving of a further dose of a drug to a patient who had previously taken the same drug and in whom an adverse event, which might be due to that drug, had subsequently occurred (Stephens, 1988). Whether accidental or deliberate, the rechallenge is generally considered a major criterion in the assessment of an adverse drug reaction. The authors administered clozapine for persistent psychiatric symptoms to patients who had previously developed NMS when treated with prior neuroleptics, mainly phenothiazines and butyrophenones, but not clozapine. It is thus misleading to suggest that clozapine has been readministered after recovery of the NMS episode when this drug has just been chosen as a further and alternative antipsychotic medication. On the other hand, the authors suggest that clozapine should be considered a drug of choice for psychotic patients with a history of NMS.

However, it has been reported that clozapine by itself may be implicated in the development of NMS (Miller et al, 1991; DasGupta \& Young, 1991). Furthermore, additional cofactors are required for the development of NMS and a number of patients may successfully tolerate careful neuroleptic reexposure after at least two weeks of complete recovery of the index episode (Pope et al, 1991). How to treat subsequent psychotic manifestations in a patient after an episode of NMS is a matter of increasing attention among clinicians, and nonneuroleptic treatments such as ECT have also been considered (Bottaï et al, 1992). Despite the distinctive pharmacological profile of clozapine among antipsychotic drugs, it may be premature to speculate about the peculiar interest of this drug in the further management of patients with a history of NMS.

Bottaï, T., Petit, P., Pinzani, V., et al (1992) Neuroleptic malignant syndrome: 11 case reports. 18 th Collegium Internationale Neuropsychopharmacologicum. Clinical Neuropharmacology. 15 (suppl. 1), 138B.

DasGuPTA, K. \& Young, A. (1991) Clozapine-induced neuroleptic malignant syndrome. Journal of Clinical Psychiatry, 52, 105-107.

Miller, D. D., Sharafuddin, M. J. \& Kathol, R. G. (1991) A case of clozapine-induced neuroleptic malignant syndrome. Journal of Clinical Psychiatry, 52, 99-101.

PoPe, H. G., AIZley, H. G., KeCk, P. E., et al (1991) Neuroleptic malignant syndrome: long-term follow-up of 20 cases. Journal of Clinical Psychiatry, 52, 208-212. 